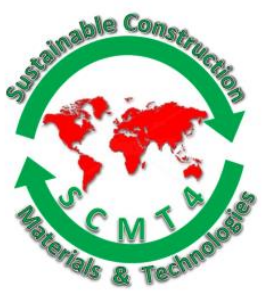

SCMT4

Las Vegas, USA, August 7-11, 2016

\title{
Simplified Modeling of Concrete Confinement
}

\author{
Gian Piero Lignola ${ }^{1 a}$, Andrea Prota ${ }^{1 b}$, and Gaetano Manfredi ${ }^{1 \mathrm{c}}$ \\ ${ }^{I}$ Department of Structures for Engineering and Architecture, University of Naples "Federico II"- \\ Naples, P. O. 80125, Italy. \\ ${ }^{1 a}$ Email: <glignola@unina.it>, ${ }^{1 b}$ Email:<aprota@unina.it>,, Email: <gamanfre@unina.it>.
}

\begin{abstract}
The goal of this research project is to model the effect of confinement on concrete members by means of externally bonded wrapping, hence to provide a simplified closed form solution to determine directly the ultimate confined concrete strength. Common cross-section shapes for reinforced concrete columns are considered herein, namely circular, solid and hollow, square and rectangular. Nowadays innovative materials are developing focusing on the sustainability of the intervention, improving "traditional" FRP materials, where the organic matrix is substituted by an inorganic matrix and fiber fabrics are substituted by biaxial grids, while almost the same fiber materials are adopted. The simplified model is practitioners and code oriented, while it is derived from a more research oriented refined iterative confinement model proposed by the same authors to evaluate the entire stress-strain relationship of confined concrete. Based on a detailed analysis of the stress state, a simplified closed form solution is proposed to account for the non-uniformly confined concrete performance exhibited in non-axisymmetric sections. The non-uniform confining stress field exhibited in such cross-sections is explicitly considered by means of the mean value integral of the pointwise variable stress state over the cross-section. The key aspect of the proposed methodology is the evaluation of the effective equivalent pressure to be inserted in a triaxial confinement model, to account for the peculiarities of square and rectangular cross-sections. Experimental data, available in the literature and representative of a wide stock of applications, were compared to the results of the theoretical simplified model to validate the proposed approach, and satisfactory results were found.
\end{abstract}

\section{INTRODUCTION}

Numerous researchers have demonstrated that circular concrete columns encounter a huge increment in strength and ductility when wrapped with FRP sheets. Square and rectangular shapes were found to encounter less increment in strength and ductility than their circular counterparts. This is on account of the uniform distribution of lateral confining pressure in circular sections, as opposed to square and rectangular counterparts, in which the confining pressure changes from a peak at the corners and diagonals, to a low value in the middle.

Existing theoretical models for foreseeing the stress-strain behavior of FRP-confined concrete are for the most part inferred for circular sections. A large portion of existing models for non circular section confinement assessment both in terms of ultimate capacity as of stress-strain relationships rely on an assumed value of an "equivalent" lateral confining pressure. 
In spite of the huge research energy in the experimental field, remarkable work is still expected to completely draw conclusions on modelling of FRP confinement.

\section{BASIS OF CONFINEMENT MODEL}

Coulomb plasticity criterion. The pioneering models studied at the beginning of last century based on Coulomb plasticity criterion (e.g. Richart et al. 1928), so they originated from solid mechanics. It was evident that Coulomb plasticity criterion was easily implemented because it accounts for a linear relationship between normalized lateral confining pressure $\mathrm{f}_{\mathrm{l}} / \mathrm{f}_{\mathrm{co}}$ and normalized confined concrete strength $\mathrm{f}_{\mathrm{cc}} / \mathrm{f}_{\mathrm{co}}$ (Eqn. 1) needing the evaluation of the $\mathrm{k}_{1}$ constant, where $\mathrm{f}_{\mathrm{co}}$ is the unconfined concrete strength.

$$
\frac{f_{c c}}{f_{c o}}=1+k_{1} \frac{f_{l}}{f_{c o}}
$$

Therefore, the tri-axial behaviour of the soil (or rock) can be assumed for the confined concrete. According to the Mohr-Coulomb failure envelop (Figure 1) under lateral confining stress $\left(\sigma_{3}\right)$, the longitudinal stress $\left(\sigma_{1}\right)$ can be evaluated as follows:

$$
\sigma_{1}=2 c \cdot \tan \left(45^{\circ}+\frac{\phi}{2}\right)+\sigma_{3} \cdot \tan ^{2}\left(45^{\circ}+\frac{\phi}{2}\right)
$$

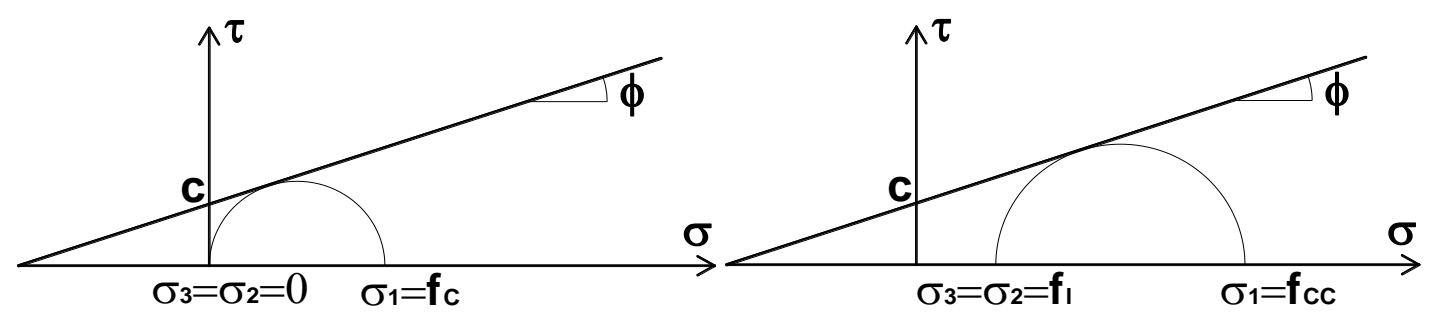

Figure 1. Unconfined and Confined Concrete strength in Mohr-Coulomb failure envelope

In Eqn. $2, \sigma_{1}$ is the longitudinal stress, $\mathrm{c}$ is the cohesion, $\sigma_{3}$ is the lateral confining stress, and $\varphi$ is the friction angle. If the stress relationship Eqn. 3 is extended to confined concrete, then $\sigma_{3}$ is the effective confinement stress, $\sigma_{3}=f_{1}$, whereas $\sigma_{1}$ is the confined concrete strength, $\sigma_{1}=f_{\text {cc. }}$ If $\sigma_{3}=0$, i.e. unconfined case, the unconfined concrete strength, $\mathrm{f}_{\mathrm{co}}$, becomes

$$
f_{c o}=2 c \cdot \tan \left(45^{\circ}+\frac{\phi}{2}\right)
$$

This physical-based constitutive model for confined concrete, Eqn. 2 can be manipulated as follows for the confined concrete (Lignola et al. 2014a):

$$
f_{c c}=f_{c o}+f_{l} \cdot \tan ^{2}\left(45^{\circ}+\frac{\phi}{2}\right)=f_{c o}+k_{1} \cdot f_{l}
$$

The friction angle of concrete usually ranges from $36^{\circ}$ to $45^{\circ}$ for typical concrete classes. The friction angle is obtained with some difficulty from the experimental tests; for that reason, the friction angle can be 
expressed as a function of concrete strength. For simplicity, $\varphi$ can be expressed as linear function of concrete strength as (Li et al. 2003)

$$
\phi=36^{\circ}+\left(f_{c o} / 35\right) \cdot 1^{\circ} \leq 45^{\circ}
$$

The value proposed by Richart et al. (1928) was $\mathrm{k}_{1}=4.1$ corresponding to $\phi=37^{\circ}$, an average value for the concrete subjected to low confinement pressure. After some triaxial tests, many authors proposed different expressions for $\mathrm{k}_{1}$. Recent studies suggest to assume $\mathrm{k}_{1}$ as a function of the confinement level and to take into account the influence of concrete strength on the ultimate behavior.

Cross section shape. The previous paragraph showed the confinement effect for concrete when a uniform pressure is applied, however typical cross sections of concrete columns are not circular, but square or rectangular (Lignola et al. 2008, 2014b, De Luca et al. 2011). The non circular shape yields to non uniform confining pressure, hence the confinement stress, $\mathrm{f}_{\mathrm{l}}$, is not easily computed for such cross sections. The aim of a simplified model, practice oriented, is to provide a reliable value of such parameter, primarily being easily estimated.

Based on a detailed analysis of the stress state, a simplified closed form solution is proposed to account for the non-uniform confined concrete behavior in non-axisymmetric sections. The non-uniform confining stress field in such cross-sections is incorporated explicitly in the model evaluating the integral mean value of the pointwise variable stress field over the cross-section. The key aspect of the proposed methodology is the evaluation of the effective equivalent pressure to be inserted in a triaxial confinement model (like as the one in Eqn. 4), to account for the peculiarities of square and rectangular cross-sections. Hence the first step is to estimate the pointwise variable stress state over the cross-section.

The passive confinement on axially loaded concrete members is due to the transverse dilation of concrete and the presence of a confining device which opposes this expansion and determines for concrete a triaxial state of stress variable point by point in a noncircular section. Braga et al. (2006) assumed that the increment of stress in the confined concrete is achieved without any out-of-plane strain (i.e. plane strain conditions). The model is based on the elasticity theory and, to quantify the forces between concrete and confinement device (Figure 2), it is necessary to estimate the expressions of parameters for some Airy's functions, ensuring compatibility and plane strain.
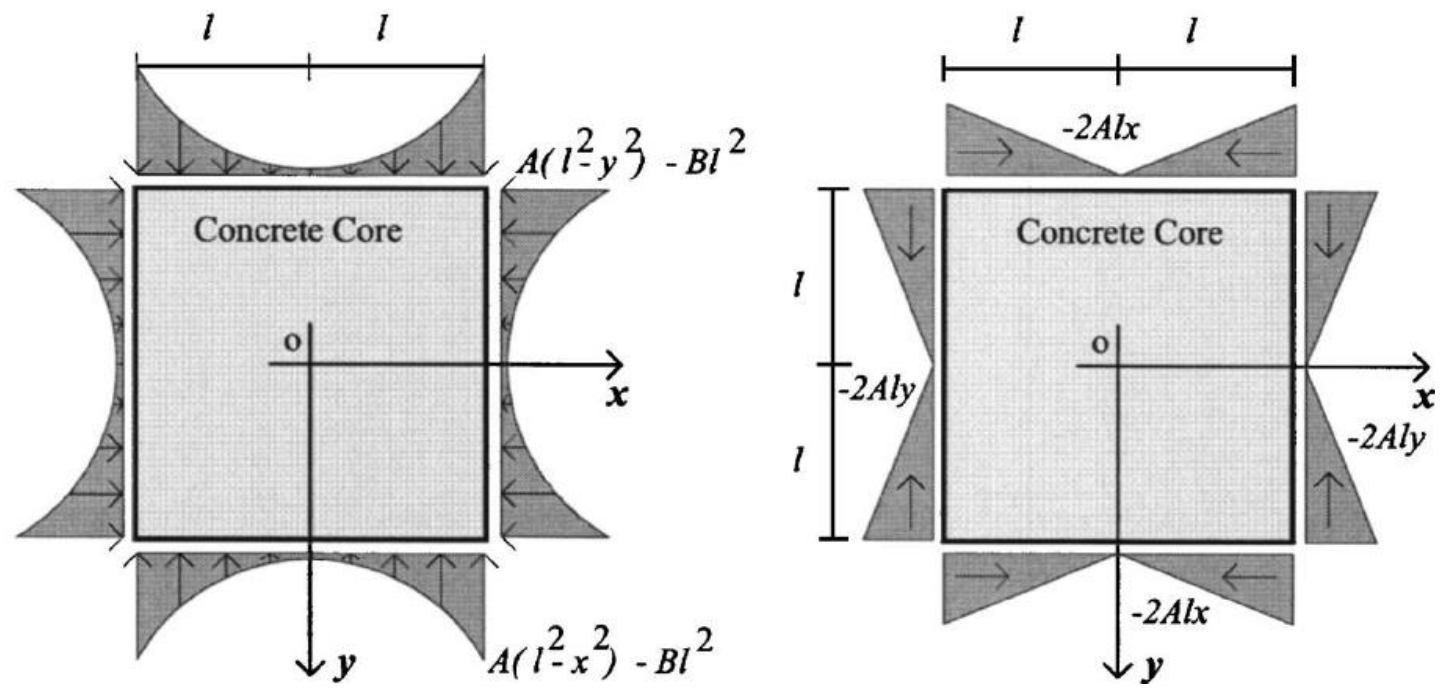

Figure 2. Confining pressures in square cross-sections 
The confinement is provided by devices without flexural stiffness (e.g. steel hoops) and extended to the case of FRP wrapping (i.e. zero flexural stiffness and thickness corresponding to the stirrup cross-section over the stirrups' spacing). The confining stress field inside the cross-section, ensuring equilibrium and compatibility, is given by the following equations:

$$
\begin{aligned}
& \sigma_{x}=A\left(x^{2}-y^{2}\right)-B l^{2} \\
& \sigma_{y}=A\left(y^{2}-x^{2}\right)-B l^{2} \\
& \tau_{x y}=-2 A x y
\end{aligned}
$$

The constants A and B have been determined (Lignola et al. 2009) by guaranteeing the compatibility of the confining device and concrete both orthogonally and parallel to the wrapping (the sum of displacements of concrete - expansion due to the axial load and contraction due to the inward confining pressure - is equal to the wrap expansion due to the same pressure, but in the outward direction).

$$
\begin{aligned}
& A=\frac{21 E_{c} E_{f} t_{f}}{25 E_{c} L^{3}+12 E_{f} t_{f} L^{2}\left(2 v_{c}+5\right)} \cdot \varepsilon_{l} \\
& B=\frac{18 E_{c} E_{f} t_{f}}{25 E_{c} L^{3}+12 E_{f} t_{f} L^{2}\left(2 v_{c}+5\right)} \cdot \varepsilon_{l}
\end{aligned}
$$

the confining pressures (Eqns. 6-8) becomes a function of axial strain $\varepsilon_{\mathrm{c}}$, in concrete. The key aspect of the model is the different contribution of confining stress field not equal in the two transverse directions $x$ and $y$.

Equivalent Confining Pressure for square cross sections. The evaluation of different contributions of confining stresses not uniform and variable in the two transverse directions allows to develop a refined model accounting for the meshing of the cross section and point wise evaluation. However it turns easily into a major disadvantage if a simplified model is expected. To gain the accuracy of the pointwise variability, but ensuring the simplicity of a practitioner oriented model, an equivalent confining pressure should be evaluated. The effective equivalent pressure when inserted in a triaxial confinement model, should transparently account for the peculiarities of square cross-section.

In non-circular cross sections, the confining pressure in two orthogonal directions $\sigma_{3}=f_{1, \min }$ and $\sigma_{2}=f_{1, \max }$, are different. Howsoever the failure envelope can be associated to the minimum confining pressure (Figure 3), being failure (outermost) circle independent on $\sigma_{2}$.

This is the main idea allowing to evaluate the equivalent confining pressure. Hence, the strength of concrete is related only to minimum confining pressure in each point (minimum principal stress, fl,min according to Eqn. 10), and integrated over the cross-section.

$$
f_{l, \text { min }}=\sigma_{3}=\frac{\sigma_{x}+\sigma_{y}}{2}-\sqrt{\frac{\left(\sigma_{x}-\sigma_{y}\right)^{2}}{4}+\tau_{x y}{ }^{2}}
$$




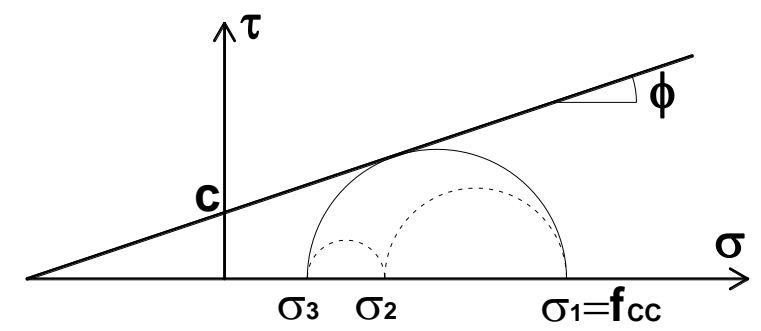

\section{Figure 3. Confined concrete strength under non uniform confining pressure field}

The axial stresses are integrated over cross-section and divided by the total area A to provide directly the average confined concrete strength, fcc,sq (see Eqn. 11). In this way an average lateral pressure (term in brackets in Eqn. 11) is evaluated, which can be assumed as the equivalent confining pressure to be inserted in the confinement model to obtain directly, and without any meshing, the confined concrete strength in square sections.

$$
\begin{array}{r}
\int_{c c, s q}=\frac{\int_{\text {Cross-Section }}\left(f_{c c}\right) d A}{\int_{\text {Cross-Section }} d A}=\frac{\int_{\text {Cross-Section }}\left(f_{c o}+k_{1} f_{l}\right) d A}{\int_{\text {Cross-Section }} d A}= \\
=f_{c o}+k_{1} \cdot\left(\frac{\int_{\text {Cross-Section }}\left(f_{l, \text { min }}\right) d A}{\int_{\text {Cection }} d A}\right)=f_{c o}+k_{1} \cdot f_{l, s q}
\end{array}
$$

and the closed form solution for equivalent confining pressure provided by the integral, is given by

$$
f_{l, s q}=\frac{4 E_{c} E_{f} t_{f}}{25 E_{c} l+12 E_{f} t_{f}\left(5+2 v_{c}\right)} \varepsilon_{l}
$$

where symbols are: $l$ the half length of a side of the square cross-section, $E_{\mathrm{f}}$ and $\mathrm{t}_{\mathrm{f}}$ the Young modulus and total thickness of the wrap respectively, $\varepsilon_{1}$ the strain in the wrap. The nonlinear mechanical properties of concrete are (secant) Young modulus $\mathrm{E}_{\mathrm{c}}$ and dilation ratio $v_{\mathrm{c}}$ at failure:

Both the nonlinear mechanical properties of concrete should be evaluated iteratively, however this makes the model not enough straightforward for practitioners. For this reason the discussion continues to account, in a simplified manner, for rectangular cross sections too and a further simplification is introduced later.

Equivalent Confining Pressure for rectangular cross sections. To provide a direct, practical tool, oriented to the profession, a simplified confinement model for rectangular cross-sections has been developed. The basic idea is that a rectangular cross-section can be seen as in between two square sections for which a reliable confinement model has been provided.

In view of this basic idea, the behavior of a rectangular cross-section is assumed as in between the behavior of two square cross-sections having the sides equal to the longest and shortest sides of the rectangular crosssection (Figure 4). According to this simplification, the lateral pressure for the rectangular cross section (having dimensions $\mathrm{L}_{\mathrm{x}} \mathrm{L}_{\mathrm{y}}$ ) is assumed to be the average of the lateral pressures for the two square cross- 
sections (having dimensions $\mathrm{L}_{\mathrm{x}}$ and $\mathrm{L}_{\mathrm{y}}$ respectively). According to this, the equivalent confining pressure for rectangular cross section is:

$$
f_{l, \text { rect }}=\left(\frac{2 E_{c} E_{f} t_{f}}{12.5 E_{c} L_{x}+12 E_{f} t_{f}\left(5+2 v_{c}\right)}+\frac{2 E_{c} E_{f} t_{f}}{12.5 E_{c} L_{y}+12 E_{f} t_{f}\left(5+2 v_{c}\right)}\right) \varepsilon_{l}
$$

Please note that the confinement equation is linear, but $E_{c}$ and $v_{c}$ are not, however the average confined concrete strength can be pragmatically obtained by inserting in the equation the average of the lateral confining stresses for the two "boundary" square cross-sections, and it becomes:

$$
f_{c c, \text { rect }}=f_{c o}+k_{1} \cdot f_{l, \text { rect }}
$$

In Eqn. 13, a geometrical reinforcement ratio, $\rho \mathrm{i}$ (where i stands for dimension along $\mathrm{x}$ or $\mathrm{y}$, and $\mathrm{Li}$ is the full side length), can be introduced as

$$
\rho_{i}=\frac{t_{f}}{l_{i}}=2 \frac{t_{f}}{L_{i}}
$$

\section{Rectangular cross section}

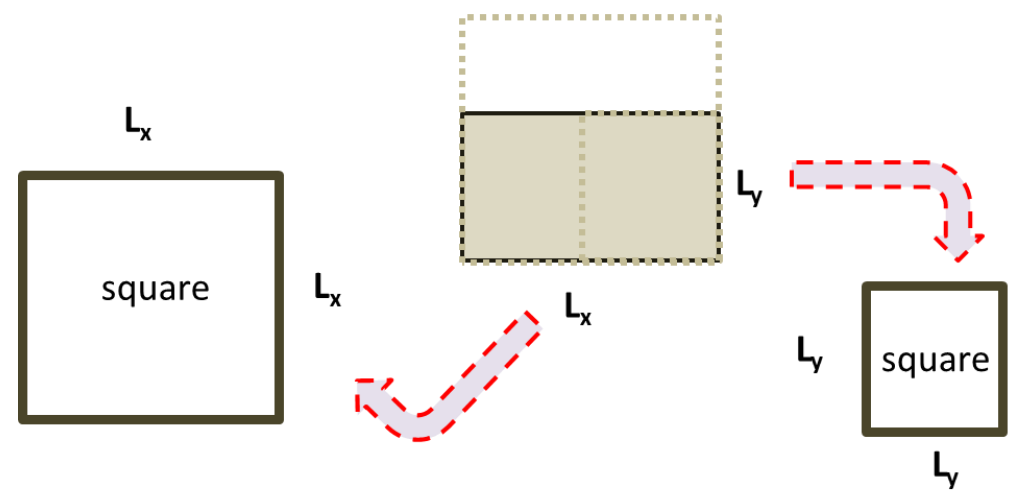

Figure 4. Basic idea for a simplified confinement model for rectangular cross-sections

Hence the equivalent confining pressure for rectangular cross section, Eqn. 13 becomes:

$$
f_{l, \text { rect }}=\left(\frac{2}{\frac{25}{\rho_{x} E_{f}}+12\left(\frac{5+2 v_{c}}{E_{c}}\right)}+\frac{2}{\frac{25}{\rho_{y} E_{f}}+12\left(\frac{5+2 v_{c}}{E_{c}}\right)}\right) \varepsilon_{l}=\left(\frac{2}{\frac{25}{\rho_{x} E_{f}}+\frac{N L}{E_{c o}}}+\frac{2}{\frac{25}{\rho_{y} E_{f}}+\frac{N L}{E_{c o}}}\right) \varepsilon_{l}
$$

where NL is the nondimensional factor accounting for the nonlinear mechanical properties of concrete (secant elastic modulus at ultimate, $\mathrm{E}_{\mathrm{c}}$, is expressed as a fraction of initial elastic Young modulus, $\mathrm{E}_{\mathrm{co}}$ ), and it makes the model not easily applicable, requiring an iterative approach. For this reason, the impact of the nondimensional factor NL can be evaluated by means of a wide sensitivity analysis based on a database of experimental tests. 


\section{PROPOSED SIMPLIFIED CONFINEMENT MODEL}

To estimate the impact of the nonlinear mechanical properties of concrete, a selection of about fifty experimental tests on rectangular (plain) concrete columns wrapped with glass and carbon FRP, and with enough data reported in relevant papers have been selected (Demers and Neale 1999, Rochette and Laboissiere 2000, Parvin and Wang 2001, Shehata et al. 2002, Chaallal et al 2003, Ilki and Kumbasar 2003, Lam et al. 2006, Ombres 2006, Micelli and Modarelli 2013). The database includes a wide stock of combinations in terms of column dimensions, concrete classes, thickness and capacity of FRP wraps.

The sensitivity analysis allowed to identify the impact of the NL term on the predictability of the model (Eqn. 14) in terms of average and coefficient of variation, $\mu$ and C.V respectively, of the ratios between theoretical predictions and experimental outcomes of confined concrete strength (to this aim, reinforced concrete columns have been discarded to avoid uncertainty related to exclusion of load carried by longitudinal reinforcement). Best performance of the model is expected when $\mu$ tends to 1 and C.V is minimum.

The expected range of NL values should account for variability of $v_{c}$ (dilation ratio at ultimate), expected in the range 0-2, and $\mathrm{E}_{\mathrm{c}}$ (secant elastic modulus at ultimate, expressed as a fraction of $\mathrm{E}_{\mathrm{co}}$ ) expected in the range 0.1-1 $\mathrm{E}_{\mathrm{co}}$, hence NL should be roughly in the range 60-1000. Figure 5 shows the outcome of this sensitivity analysis, and it is quite clear that a value of $\mathrm{NL}=0$ yields to the best performance of the model and simplifies drastically the model, too. However it is not an unexpected result because even if range of $\mathrm{NL}$ seems quite high, yet in the worst case (i.e. higher $\mathrm{NL}$ ), the term $\mathrm{NL} / \mathrm{E}_{\mathrm{co}}$ is negligible (i.e. at least one or two orders of magnitude lower, but usually more) compared to $25 /\left(\rho_{\mathrm{i}} \mathrm{E}_{\mathrm{f}}\right)$.

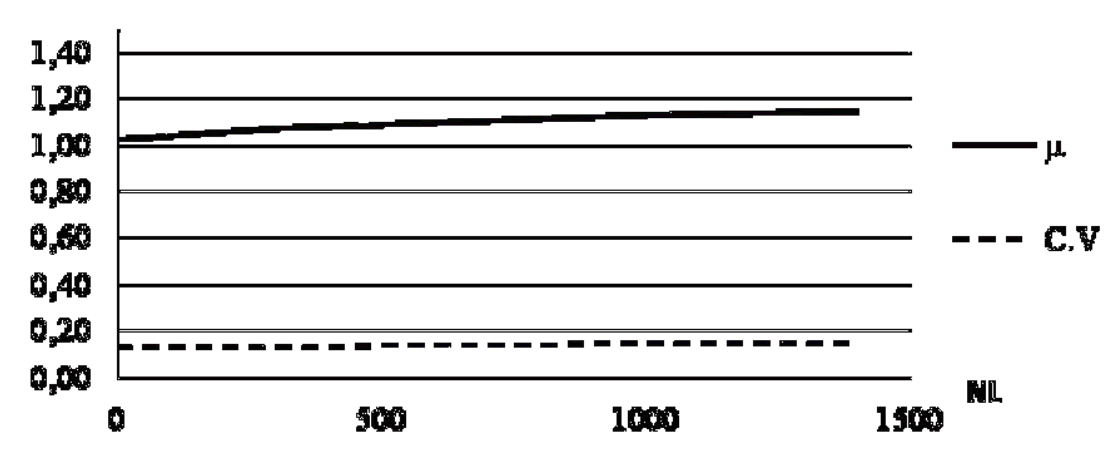

Figure 5. Sensitivity analysis on parameter NL

In Eqn. 13, a further simplification along with the assumption of $\mathrm{NL}=0$ can be provided by introducing a mechanical reinforcement ratio, $\omega_{\mathrm{i}}$ (where i stands for dimension along $\mathrm{x}$ or $\mathrm{y}$ ), as:

$\omega_{i}=\frac{E_{f} \varepsilon_{l} t_{f}}{f_{c o} l_{i}}=2 \frac{E_{f} \varepsilon_{l} t_{f}}{f_{c o} L_{i}}=\frac{E_{f} \varepsilon_{l}}{f_{c o}} \rho_{i}$

Hence the final simplified confinement equation for rectangular cross section, Eqn. 14 becomes:

$$
\frac{f_{c c, r e c t}}{f_{c o}}=1+k_{1} \frac{f_{l, r e c t}}{f_{c o}}=1+\frac{2 k_{1}}{25}\left(\omega_{x}+\omega_{y}\right)
$$


An overview of the predictability of the proposed simplified model is provided in Figure 6 on the wellknown format of the $45^{\circ}$ line. It is noted that the model is generally not conservative, however the predictability is quite high (point cloud aligns along $45^{\circ}$ line) and the definition of partial safety factors, for design purposes, requires a further step (i.e. a statistical regression based calibration, like as in Lignola et al. 2014c, to account for all sources of uncertainties, apart the model equation) out of the scope of present work.

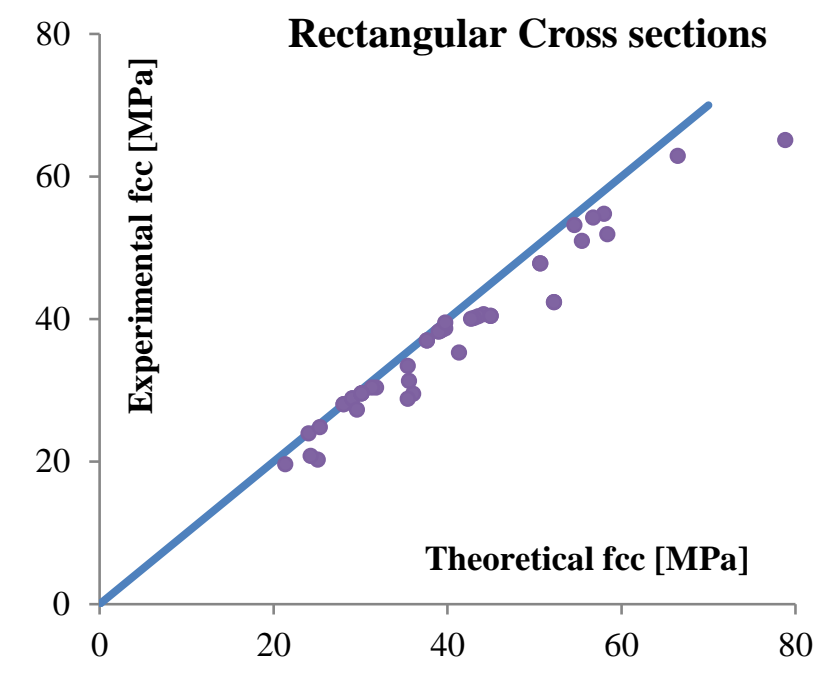

\section{Figure 6. Validation of the proposed model on experimental database of confined concrete strengths, $\mathbf{f}_{\mathrm{cc}}$}

It is worth noting that in "classical" approaches (e.g. CNR DT200R1 2013, one of the most recent) the lateral pressure is evaluated according to an equivalent circular cross section having a diameter equal to the circle inscribed or circumscribed to the real cross section and reduced by a "conventional" shape factor, $\mathrm{k}_{\mathrm{e}}$. For instance, compared to CNR DT200R1 formulation (right hand side of Eqn. 18, "conventional" approach based on equivalent circular section), the proposed model (right hand side of Eqn. 18, mechanically based accounting for pointwise variability of confining pressure and simplified) provides a $\mathrm{k}_{\mathrm{e}}=0.16$. However the "conventional" models are commonly based on empirical best fitting; hence in the CNR DT200R1 case, $\mathrm{k}_{1}=2.6$ and lateral pressure normalized by $\mathrm{f}_{\text {co }}$ is to the power of $2 / 3$, so they represent further differences with proposed model.

$$
f_{l, r e c t}=k_{e} \frac{L_{x}+L_{y}}{L_{x} L_{y}} t_{f} E_{f} \varepsilon_{l}=\frac{2}{25}\left(\omega_{x}+\omega_{y}\right) f_{c o}
$$

Finally the simplified confinement equation for rectangular cross section, Eqn. 17, can be pragmatically extended to the case of square cross sections where it can be assumed that $\omega_{\mathrm{x}}=\omega_{\mathrm{y}}=\omega$ (or similarly $\mathrm{L}_{\mathrm{x}}=\mathrm{L}_{\mathrm{y}}=\mathrm{L}$ ), hence:

$$
\frac{f_{c c, s q}}{f_{c o}}=1+k_{1} \frac{f_{l, s q}}{f_{c o}}=1+\frac{4 k_{1}}{25} \omega
$$




\section{CONCLUSION}

The aim of this work is to effectively model the confinement of non-circular members accounting for a mechanically based approach. However the pointwise great variability of stresses and the nonlinear mechanical behavior of confined material (i.e. concrete) makes the modelling very complex and requiring at least iterative evaluations and probably a computational support.

However to provide a simplified closed form solution to determine directly the ultimate confined concrete strength (even by hand calculation), starting from a detailed description of the confining stress field, a series of sequential simplifications were provided.

Based on the accuracy of the expected result, a practitioner could stop at every "simplification" step, but the last step provides a very simple equation yet providing satisfactory results. The most simple equation is analogous to those provided by "conventional" models (empirically, best fitting, based), however the derivation is quite different, more rigorous for square cross sections, more approximated for rectangular cross sections, yet effective.

\section{ACKNOWLEDGEMENTS}

The analyses were developed within the activities of Rete dei Laboratori Universitari di Ingegneria Sismica - ReLUIS for the research program funded by the Dipartimento di Protezione Civile - Progetto Esecutivo 2014-2018.

\section{REFERENCES}

Braga F, Gigliotti R and Laterza M (2006) "Analytical Stress-strain relationship for concrete confined by steel stirrups and/or FRP jackets". J. Struct. Eng., 132(9), 1402-1416.

Chaallal O, Shahawy M and Hassa M (2003) "Performance of axially loaded short rectangular columns strengthened with carbon FRP wrapping” J. Compos. Constr., 7(3), 200-208.

CNR-DT 200 R1 (2013), Guide for the Design and Construction of Externally Bonded FRP Systems for Strengthening Existing Structures, Italian Council of Research (CNR), Rome (Italy): 1-167.

De Luca A, Nardone F, Matta F, Nanni A, Lignola GP and Prota A (2011) "Structural Evaluation of FullScale FRP-Confined Reinforced Concrete Columns" J. Compos. for Construction 15(1), 112-123.

Demers M and Neale KW (1999) "Confinement of reinforced concrete columns with fiber-reinforced composite sheets-an experimental study". Can. J. Civ. Eng., 26, 226-241.

Ilki A and Kumbasar N (2003) "Compressive behavior of carbon fiber and not circular cross-section", Journal of earthquake engineering, 7(3), 381-406.

Lam L, Teng JG, Cheung CH and Xiao Y (2006) "FRP-confined concrete under cyclic axial compression". Cem Concr Compos. 28, 949-958.

Li Y, Lin C, and Sung Y (2003) "A Constitute model for concrete confined with carbon fibre reinforced plastics". Mech. Mater., 35, 603-619.

Lignola GP, Angiuli R, Prota A and Aiello MA (2014a) "FRP Confinement of masonry: analytical modeling”. Materials and Structures. 47(12), 2101-2115.

Lignola GP, Jalayer F, Nardone F, Prota A and Manfredi G(2014c) "Probabilistic design equations for the shear capacity of RC members with FRP internal shear reinforcement". Composites: Part B, 67, 199208. 
Lignola GP, Prota A and Manfredi G (2014b) "Simplified Modeling of Rectangular Concrete CrossSections Confined by External FRP Wrapping” Polymers 6(4), 1187-1206.

Lignola GP, Prota A, Manfredi G and Cosenza E (2009) "Analysis of FRP Confinement On Prismatic RC Columns". Proceedings of 9th International Symposium on Fiber Reinforced Polymer Reinforcement for Concrete Structures FRPRCS9 2009. Sydney, Australia, \#129.

Micelli F and Modarelli R (2013) "Experimental and analytical study on properties affecting the behavior of FRP-confined concrete" Composites Part B, 45(1), 1420-1431.

Ombres L (2006) "Influenza del Sistema di rinforzo sul confinamento di elementi compressi in calcestruzzo rinforzato con FRP”. Atti del $16^{\circ}$ Congresso C.T.E. Parma, in Italian.

Parvin A and Wang W (2001) "Behaviour of FRP Jacked Concrete Columns Under Eccentric Loading", Journal of Composites for Construction, 5(3), 146-152.

Richart FE, Brandtzaeg A and Brown RL (1928) "A Study of the Failure of Concrete Under Combined Compressive Stresses" Univ. of Illinois Engineering Experimental Station, Champaign, Ill, Bulletin 185.

Rochette P and Labossière P (2000) "Axial testing of rectangular column models confined with composites". J Compos Constr, 4(3), 129-136.

Shehata IAEM, Carneiro LAV and Shehata LCD (2002) "Strength of confined short concrete columns. Confined whit CFRP sheet" Materials and structures, 35(1), 50-58. 